\title{
Comparação entre a Relação Neutrófilo-Linfócito Precoce e Tardia na Predição de Eventos Adversos em Pacientes com IAMCSST submetidos à ICP Primária
}

\author{
Early vs. Late Neutrophil-To-Lymphocyte Ratio for the Prediction of Adverse Outcomes in Patients with STEMI \\ Undergoing Primary PCI
}

\author{
Guilherme Pinheiro Machado, ${ }^{1 \oplus}$ Gustavo Neves de Araujo, ${ }^{2}$ Daniele Maltauro, ${ }^{1 \oplus}$ Julia Custodio, ${ }^{1}$ Victoria Milan, ${ }^{3}$ \\ Marco Wainstein ${ }^{2}$ \\ Universidade Federal do Rio Grande do Sul, ${ }^{1}$ Porto Alegre, RS - Brasil \\ Hospital de Clínicas de Porto Alegre, ${ }^{2}$ Porto Alegre, RS - Brasil \\ Universidade Federal de Ciências da Saúde de Porto Alegre Faculdade de Medicina, ${ }^{3}$ Porto Alegre, RS - Brasil
}

\section{Introdução}

A relação neutrófilo-linfócito (NLR) na admissão hospitalar já se mostrou capaz de prever eventos adversos em pacientes com infarto agudo do miocárdio com supra desnível do segmento ST (IAMCSST).1-3 Evidências recentes demonstraram que a NLR continua aumentando no período de 48 a 72 horas nos pacientes que apresentam resultados piores.4 Portanto, nosso objetivo foi comparar a capacidade prognóstica da NLR na admissão e tardia para eventos adversos em pacientes com IAMCSST submetidos à intervenção coronária percutânea primária (ICPp).

\section{Métodos}

Este foi um estudo coorte prospectivo com pacientes consecutivos admitidos com IAMCSST que passaram por ICPp e foram acompanhados durante 12 meses. A NLR foi calculada dividindo-se o número de neutrófilos pelo número de linfócitos obtidos da mesma amostra sanguínea. A NLR foi avaliada na admissão e no período de 48 a 72 horas após o procedimento (NLR tardia) como parte do tratamento de rotina. Outros detalhes sobre informações procedimentais, coleta de dados, definições clínicas, critérios de exclusão e diretrizes éticas estão descritos em outros locais. ${ }^{2}$ A NLR alta foi definida como acima do tercil superior. A análise da curva de característica de operação do receptor (ROC) foi realizada para calcular a área sob a curva (AUC) para a ocorrência de mortalidade em curto e longo prazo e de eventos cardíacos adversos maiores (MACE) Foram realizadas análises multivariadas pela regressão de Poisson com variância robusta para avaliar o valor preditivo independente da NLR tardia. Para o modelo multivariado, os fatores de risco que

\section{Palavras-chave}

Infarto do Miocárdio; Angioplastia; Mortalidade; relação neutrófilo-linfócito; Inflamação; Fatores de Risco.

Correspondência: Guilherme Pinheiro Machado •

Hospital de Clinicas de Porto Alegre - Rua Domingos Crescencio, 545. CEP

90650-090, Porto Alegre, RS - Brasil

E-mail: gpmachado89@gmail.com

Artigo recebido em 14/04/2020, revisado em 16/08/2020, aceito em 09/09/2020

DOI: https://doi.org/10.36660/abc.20200327 foram preditores univariados ( $p<0,05$ ) foram considerados inicialmente como fatores ou covariáveis. As análises de concordância foram comparadas pelo teste de De Long, enquanto os métodos de Kaplan-Meier foram comparados com testes de Log-Rank, realizados utilizando-se o software MedCalc Statistical, versão 14.8.1 (MedCalc Software, Ostend, Bélgica). Todas as demais análises estatísticas foram realizadas utilizando-se o software SPSS Statistics for Windows, v.21.0. (IBM Corp., Armonk, NewYork, EUA).

\section{Resultados}

Entre março de 2011 e dezembro de 2018, 864 pacientes compareceram à nossa instituição, diagnosticado com IAMCSST, e 779 deles foram incluídos na análise. A média de idade foi de 60,68 ( \pm 12$), 66,4 \%$ dos pacientes eram do sexo masculino, 62,1\% tinham hipertensão e $24 \%$ tinham diabetes.

Na análise multivariada, quando ajustada por idade, tempo dor-porta, doença renal crônica prévia, infarto do miocárdio (IM) prévio, hipotensão na admissão, acesso femoral, tempo de fluoroscopia, volume de contraste, classificação de trombose no infarto do miocárdio (TIMI), fração de ejeção do ventrículo esquerdo $\leq 40 \%$ antes da alta, a NLR continuou sendo um preditor independente da mortalidade hospitalar, MACE hospitalar e mortalidade após 1 ano (risco relativo $[R R]=14,9,95 \%$ intervalo de confiança $[95 \% I C]=3,4-$ $80,35, p=0,001 ; R R=3,4,95 \% I C=1,2-9,1, p=0,01$; $R R=7,6,95 \% I C=2,9-26,1, p=0,01$, respectivamente). O uso da NLR tardia aumentou significativamente a AUC de mortalidade hospitalar de 0,55 para 0,84 (Sensibilidade 81,2\%, Especificidade 75,6\%, Valor preditivo positivo 24,5 e Valor preditivo negativo 97,7$)$. Os dados discriminados dos demais resultados estão descritos na Figura 1. Ao final de 1 ano de acompanhamento, o índice de mortalidade global foi de $28,6 \%$ no grupo de NLR alta, hazard ratio $(H R)=3,07$ $(95 \%$ IC $=1,9-4,8) ; p<0,0001$; Figura 2).

\section{Discussão}

No presente estudo coorte de pacientes de IAMCSST que passaram por ICPp, a NLR tardia foi fortemente associada à mortalidade no curto e no longo prazo e aos MACE. Além disso, a NLR tardia aumenta a capacidade da NLR na admissão de eventos adversos nesses pacientes. Até onde sabemos, 


\section{Carta Científica}

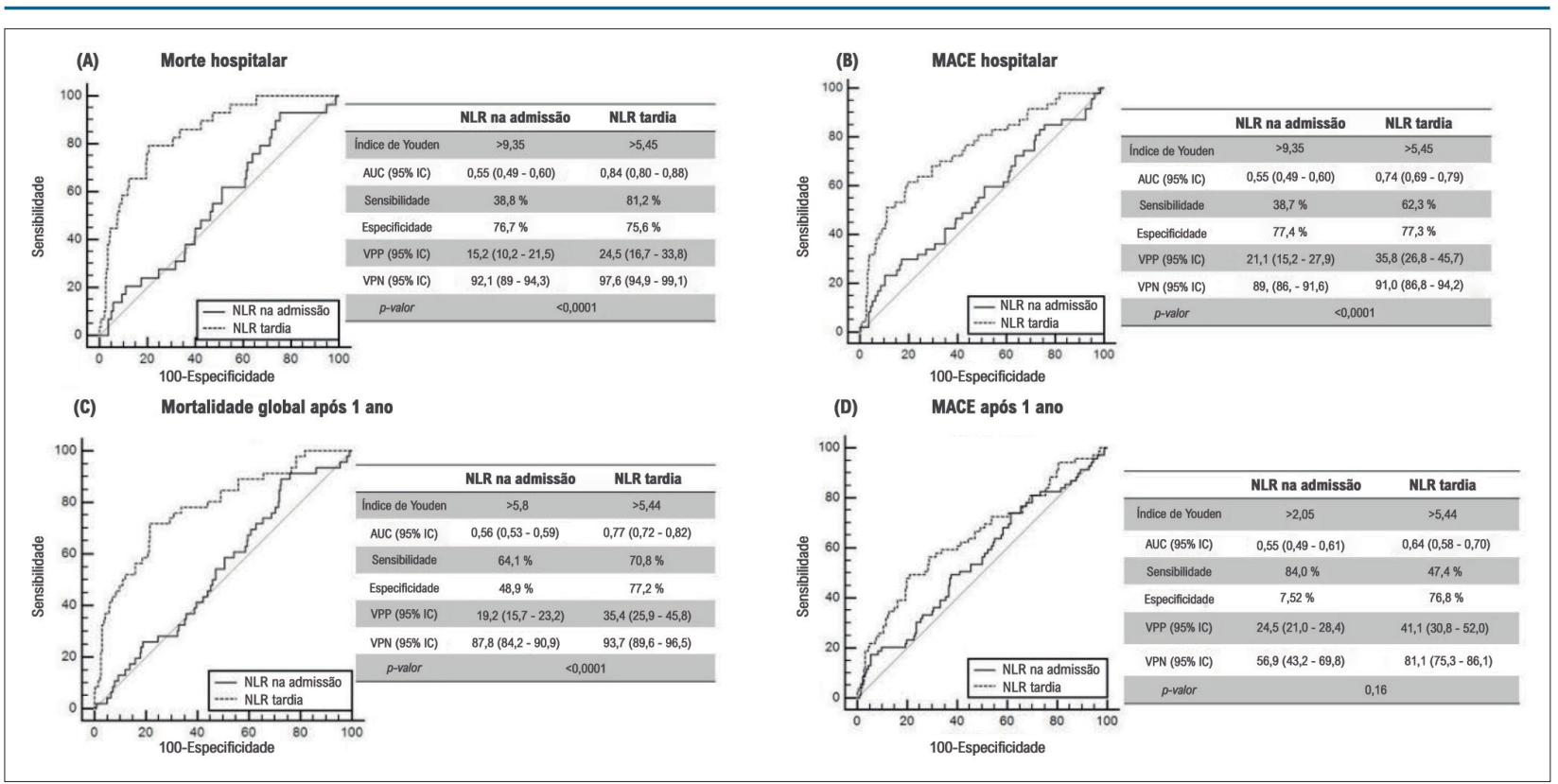

Figura 1 - Gráfico de característica de operação do receptor (ROC) mostrando as áreas sob acurva (AUC) da relação neutrófilo-linfócito (NLR) na admissão e NLR tardia para (A) mortalidade hospitalar, (B) eventos cardiovasculares maiores hospitalares (MACE), (C) mortalidade global após 1 ano e (D) MACE após 1 ano.

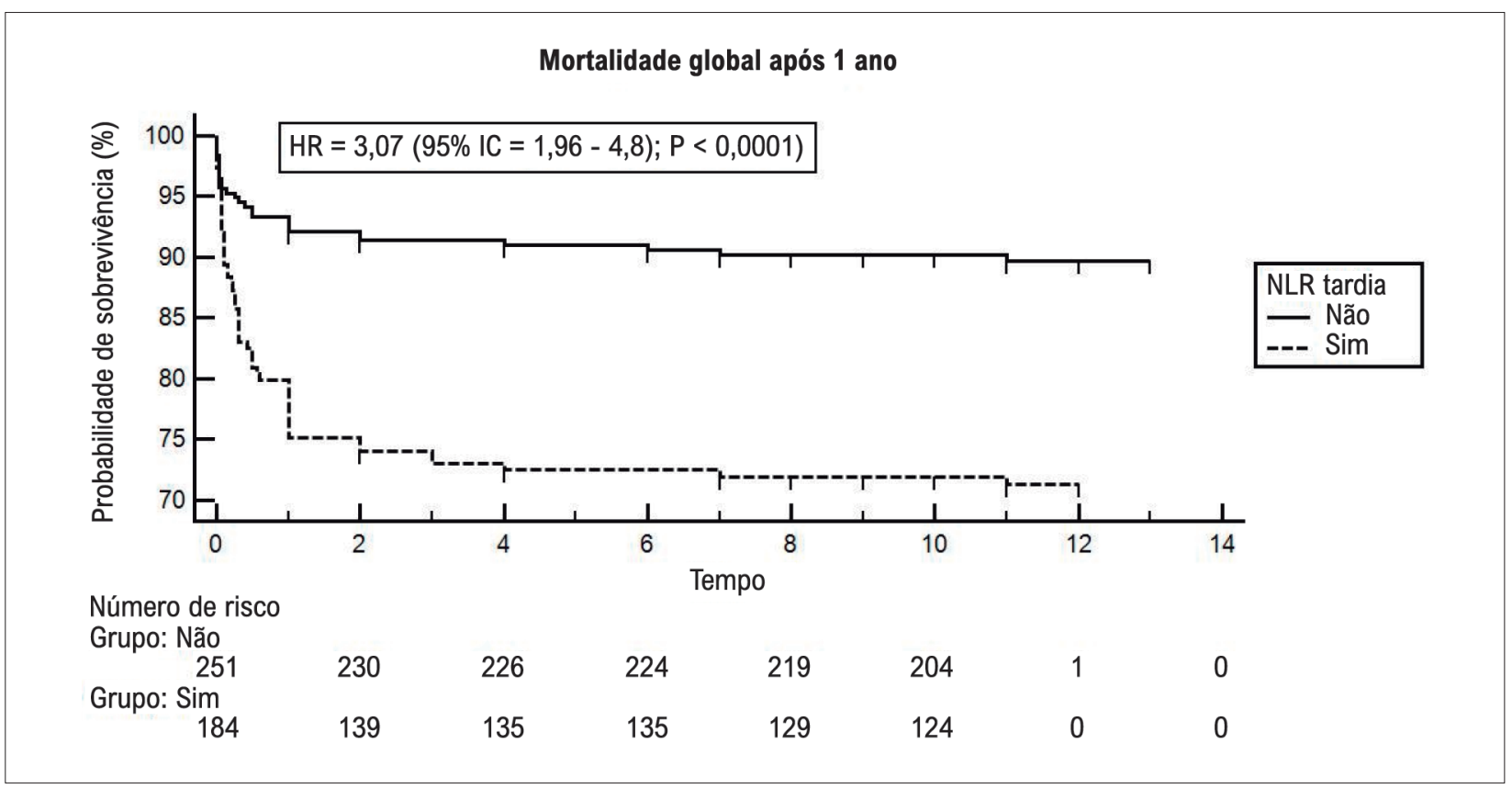

Figura 2 - Curvas tempo-evento para mortalidade global após 1 ano de relação neutrófilo-linfócito (NLR). Os índices de eventos foram calculados utilizando-se os métodos de Kaplan-Meier, e comparados com uso do teste Log-rank

esta foi a primeira vez em que a NLR tardia foi avaliada consistentemente nesse cenário.

A distribuição normal da NLR ainda exige debates. Forget et al., ${ }^{5}$ estudaram indivíduos saudáveis, e os valores variaram entre 0,78 e 3,5, permanecendo estáveis após 48 horas. Recentemente, Kim et al., ${ }^{6}$ observaram que a NLR aumenta ao longo do tempo em indivíduos com doença cardiovascular, atingindo os valores de pico próximo ao momento de um evento adverso. Um estudo recente demonstrou que os pacientes que experimentaram resultados adversos durante o período de acompanhamento tiveram um aumento agudo dos valores de NLR 48 horas após o procedimento. ${ }^{4}$ Esses resultados corroboram os achados de Kim et al., ${ }^{6}$ descritos acima. 
No presente estudo, quando os valores de NLR tardia foram usados para avaliar a capacidade de prever eventos adversos, houve um aumento significativo na $A \cup C$ quando comparada à NLR na admissão. Isso pode ser explicado porque os neutrófilos são os primeiros leucócitos a infiltrarem o miocárdio infartado, liberando uma variedade enzimas proteolíticas que causam ruptura de placa, expansão do infarto, ativação da via da coagulação, e instabilidade elétrica cardíaca. ${ }^{7-9}$ Além disso, há evidências do prolongamento da vida de neutrófilos em placas instáveis. ${ }^{10} \mathrm{Em}$ contraste com o aumento de neutrófilos nas áreas lesionadas do miocárdio, houve redução dos linfócitos devido ao aumento dos níveis de cortisol, catecolaminas e citocinas pró-inflamatórias no IAMCSST. ${ }^{11,12}$ Isso sugere que a resposta inflamatória exacerbada após o evento define os piores resultados para esses pacientes.

Na prática clínica da maioria dos centros mundiais, a contagem de leucócitos é realizada rotineiramente durante a hospitalização por evento coronário agudo. No presente estudo, uma medição de NLR no período de 48 a 72 horas foi um preditor forte dos resultados adversos, o que destaca a possível aplicação desses marcadores inflamatórios acessíveis e prontamente disponíveis para a estratificação de risco após o infarto do miocárdio.

\section{Contribuição dos autores}

Concepção e desenho da pesquisa: Machado GP, Maltauro D, Custodio J, Milan V; Obtenção de dados: Maltauro D,
Custodio J, Milan V; Análise e interpretação dos dados e Análise estatística: Machado GP, Araujo GN; Redação do manuscrito: Machado GP, Araujo GN, Wainstein M; Revisão crítica do manuscrito quanto ao conteúdo intelectual importante: Araujo GN, Wainstein M.

\section{Potencial conflito de interesses}

Declaro não haver conflito de interesses pertinentes.

\section{Fontes de financiamento}

O presente estudo não teve fontes de financiamento externas.

\section{Vinculação acadêmica}

Este artigo é parte de tese de Doutorado de Guilherme Pinheiro Machado pela Universidade Federal do Rio Grande do Sul.

\section{Aprovação ética e consentimento informado}

Este estudo foi aprovado pelo Comitê de Ética do Hospital de Clinicas de Porto Alegre sob o número de protocolo 2018/0436. Todos os procedimentos envolvidos nesse estudo estão de acordo com a Declaração de Helsinki de 1975 , atualizada em 2013. O consentimento informado foi obtido de todos os participantes incluídos no estudo

\section{Referências}

1. Park JJ, Jang H-J, Oh I-Y, Yoon C-H, Suh J-W, Cho Y-S, et al. Prognostic value of neutrophil to lymphocyte ratio in patients presenting with ST-elevation myocardial infarction undergoing primary percutaneous coronary intervention. Am J Cardiol. 2013;111(5):636-42.

2. Pinheiro Machado G, Araujo GN, Carpes CK, Lech MC, Mariani S, Valle $\mathrm{FH}$, et al. Elevated neutrophil-to-lymphocyte ratio can predict procedural adverse events in patients with ST-elevation myocardial infarction undergoing primary percutaneous coronary intervention. Coron Artery Dis . 2019;30(11):20-5.

3. Machado GP, Araujo GN, Carpes CK, Lech M, Mariani S, Valle FH, et al. Comparison of neutrophil-to-lymphocyte ratio and mean platelet volume in the prediction of adverse events after primary percutaneous coronary intervention in patients with ST-elevation myocardial infarction. Atherosclerosis. 2018;274:212-7.

4. Machado GP, Araujo GN, Carpes CK, Niches M, Fracasso JF, Custodio $J \mathrm{~L}$, et al. Temporal pattern of neutrophil-to-lymphocyte ratio in patients with ST-elevation myocardial infarction undergoing primary percutaneous coronary intervention. Coron Artery Dis. 2019 Dec;30(8):631-3.

5. Forget $\mathrm{P}$, Khalifa C, Defour J-P, Latinne D, Van Pel M-C, De Kock M. What is the normal value of the neutrophil-to-lymphocyte ratio? BMC Res Notes. 2017;10(1):12.
6. Kim S, Eliot M, DC K, Wu W, KT K. Association of neutrophil-to-lymphocyte ratio with mortality and cardiovascular disease in the jackson heart study and modification by the duffy antigen variant. JAMA Cardiol

7. Tanriverdi Z, Colluoglu T, Dursun H, Kaya D. The Relationship between neutrophil-to-lymphocyte ratio and fragmented QRS in acute STEMI patients treated with primary PCl. J Electrocardiol. $2017 \mathrm{Nov}$;50(6):876-83.

8. Arruda-Olson AM, Reeder GS, Bell MR, Weston SA, Roger VL. Neutrophilia predicts death and heart failure after myocardial infarction: A communitybased study. Circ Cardiovasc Qual Outcomes. 2009;2(6):656-62.

9. Madjid M, Awan I, Willerson JT, Casscells SW. Leukocyte count and coronary heart disease: implications for risk assessment. J Am Coll Cardiol. 2004 Nov; 44(10):1945-56.

10. Narducci ML, Grasselli A, Biasucci LM, Farsetti A, Mule A, Liuzzo G, et al. High telomerase activity in neutrophils from unstable coronary plaques. J Am Coll Cardiol. 2007 Dec;50(25):2369-74.

11. Nunez J, Sanchis I, Bodi V, Nunez E, Mainar L, Heatta AM, et al. Relationship between low lymphocyte count and major cardiac events in patients with acute chest pain, a non-diagnostic electrocardiogram and normal troponin levels. Atherosclerosis. 2009 Sep;206(1):251-7.

12. Onsrud M, Thorsby E. Influence of in vivo hydrocortisone on some human blood lymphocyte subpopulations. I. Effect on natural killer cell activity. Scand J Immunol. 1981;13(6):573-9. 